\title{
BORNAND Sandra \& DERIVE Jean (dir.). - Les canons du discours et la langue: Parler juste
}

\author{
Nathaniel Gernez
}

\section{OpenEdition}

Journals

Édition électronique

URL : https://journals.openedition.org/etudesafricaines/31906

DOI : 10.4000/etudesafricaines.31906

ISSN : $1777-5353$

\section{Éditeur}

Éditions de l'EHESS

\section{Édition imprimée}

Date de publication : 17 septembre 2020

Pagination : $710-713$

ISBN : 978-2-7132-2829-2

ISSN : 0008-0055

\section{Référence électronique}

Nathaniel Gernez, «Bonnand Sandra \& Derive Jean (dir.). - Les canons du discours et la langue : Parler juste », Cahiers d'études africaines [En ligne], 239 | 2020, mis en ligne le 17 septembre 2020, consulté le 06 janvier 2023. URL : http://journals.openedition.org/etudesafricaines/31906 ; DOI : https://doi.org/ 10.4000/etudesafricaines.31906

Ce document a été généré automatiquement le 6 janvier 2023.

Tous droits réservés 


\title{
BORNAND Sandra \& DERIVE Jean (dir.). - Les canons du discours et la langue: Parler juste
}

\author{
Nathaniel Gernez
}

\section{RÉFÉRENCE}

BORNAND Sandra \& DERIVE Jean (dir.). - Les canons du discours et la langue : Parler juste.

Paris, Karthala, 2018, 328 p.

1 Que peut nous apporter la notion de «canons du discours"? Le concept de canon renvoie communément aux caractéristiques formelles d'un genre de la littérature orale ou écrite. En l'associant au discours, Sandra Bornand et Jean Derive proposent une définition plus vaste et plus stimulante intellectuellement: il s'agit d'« un concept relatif qui définit un idéal de performance en fonction des contingences variables de la communication» (p.12). Il ne s'agit donc pas d'étudier les canons comme des règles abstraites, figées, mais comme des règles qui prennent sens en contexte et qui influent sur la prise de parole. Le lien entre le titre et le sous-titre s'éclaire: l'idéal de performance, qui peut être régi par des discours métapragmatiques ou des règles implicites, liés aux attentes sociales et au contexte d'énonciation, conditionne le «parler juste».

2 Pour cette première approche de la notion de canons du discours, les auteurs de l'ouvrage proposent de l'appréhender à partir de formes énonciatives appartenant à des « genres du discours » bien définis. La diversité des contextes en Afrique mais aussi la diversité des genres (poésies, chansons, contes, romans, films, insultes, sms) et des disciplines concernées (anthropologie, anthropologie linguistique, ethnomusicologie, littérature, linguistique) montrent que la notion de canons du discours suscite un renouvellement des questionnements dans de nombreux domaines. 
Ces questionnements s'inscrivent indubitablement dans le sillage de Dell Hymes ${ }^{1}$ et de ce que celui-ci nomme la " compétence de communication » : savoir quand et comment parler de façon appropriée en fonction du contexte. Toutefois, en y adjoignant la notion de canons du discours, il me semble que les auteurs de l'ouvrage proposent une analyse du «parler juste» qui n'est plus seulement considéré comme une compétence personnelle (individuelle) mais comme une construction sociale qui nécessite de prendre en considération les influences multiples et réciproques entre l'énonciateur, les règles canoniques et l'auditoire.

4 Le premier chapitre en propose un exemple très convaincant. Katell Morand analyse ce qui fait la «justesse» lors de performances de poésie chantée en amharique. Son approche de la poésie à partir d'observations en situation lui permet, tout d'abord, de redéfinir ce qui avait été considéré, à partir de transcriptions écrites, comme les canons $\mathrm{du}$ genre. Elle montre ensuite que le poète cherche à susciter des conditions émotionnelles particulières. Sa maitrise conforme des intonations et des mouvements mélodiques propres à susciter un sentiment d'ardeur lui permettent de galvaniser ceux qui l'écoutent, tandis qu'il joue avec le public en exacerbant son attente de la dernière phrase du distique. D'ailleurs, ce n'est qu'une fois cette dernière phrase prononcée, à condition que le poète ait réussi à délivrer une affirmation personnelle, une conviction profonde qui l'implique, lui et son auditoire, que sa performance aura atteint la « justesse » désirée.

5 Comme précisé en introduction, les règles canoniques ne sont pas immuables. Les contributions de Graham Furniss (chapitre 2) et de Françoise Ugochukwu (chapitre 3) montrent justement comment les pratiques et les évaluations du public peuvent contribuer à façonner les canons. Ainsi, au chapitre 2, les débats entre intellectuels, mais également entre lecteurs, sur les qualités et les défauts d'une littérature populaire en hausa, apparus dans les années 1990 à Kano (Nigéria), contribuent à valoriser ou à délégitimer ce nouveau canon. Les lecteurs et les critiques s'interrogent sur la conformité des thèmes abordées avec les valeurs de l'islam ou les «traditions hausa » et sur la réaction que devrait avoir l'État vis-à-vis de ces productions: censure ou suppression. À l'inverse, les formes canoniques du film igbo (Nollywood), étudiées au chapitre 3, se constituent en héritant des canons de la littérature orale. En effet, l'improvisation des acteurs, la centralité de la parole (par rapport à l'action), le recours à des personnages stéréotypés, l'alternance entre parole, musique et chant, ainsi que la visée pédagogique des films, sont autant d'aspects qui sont empruntés à la littérature orale. Ces formes héritées et adaptées au cinéma confèrent une "patte " particulière aux films de Nollywood que le public souhaite retrouver et qui est toujours prégnante dans les productions plus récentes disposant des évolutions techniques et de budgets plus conséquents.

6 Awa Traoré et Jean Derive considèrent les règles canoniques comme une trame garantissant à la fois la possibilité d'improvisation de l'énonciateur et la nécessité de respecter un certain nombre de passages obligés. Ils le montrent à partir d'une analyse des contes dioula (parler de Kong). Les formules que l'on retrouve à différents moments du récit, notamment au début et à la fin, transmettent aussi un discours sur le statut du conte dans la société des Dioula de Kong: le conte est indépendant, il appartient au patrimoine collectif, le conteur ne se l'approprie que temporairement, dans une performance singulière qui lui est propre et dont il a l'entière responsabilité. 
7 Les canons du discours sont parfois liés à " une exigence canonique d'ordre social » (p. 126) concernant les enjeux de la parole et le statut de l'énonciateur. Samia Khichane (chapitre 5) montre qu'en Kabylie, l'injure réussie nécessite un savant dosage : il faut qu'elle soit suffisamment intelligible et pertinente pour faire mouche, sans être ni trop directe ni trop vulgaire pour ne pas déshonorer celui ou celle qui la profère. Ainsi, dans le cadre de l'énonciation féminine, le modèle idéal de l'injure se présente sous la forme d'une maxime ou d'un proverbe comportant un sous-entendu qui fait sens dans la situation de communication. Ces propos s'adressent à une injuriaire mais visent une tierce personne présente, l'injuriée. Cette forme d'injure permet de respecter les canons d'efficacité tout en préservant son honneur.

Les analyses de Catherine Taine-Cheikh sur les canons de la poésie maure montrent que se conformer aux règles canoniques permet aussi de les renforcer et de faire perdurer certains traits linguistiques. Ce genre est évidemment pourvu de règles strictes, mais leur permanence et leur conformité, tout à fait remarquables, participent à la grande stabilité de la langue ḥassāniyya.

9 Les chapitres qui suivent pourraient être considérés comme un deuxième temps de l'ouvrage, dans lequel il est davantage question de la réappropriation et de la transformation des formes canoniques.

10 Trois contributions explorent l'influence que peut avoir un énonciateur de génie sur les règles canoniques. Sebastien Boulay (chapitre 7) s'intéresse à un poème satirique de Bechir Ould 'Ely critiquant un transfuge du Front Polisario au Sahara occidental. Transgressif, mais particulièrement bien réalisé, les formes de ce poème ont été pérennisées par sa diffusion sur Internet. Repris maintes fois dans des montages vidéo qui renforcent la critique, il fut également l'occasion d'une réponse sous forme de pastiche. Le contenu polémique de ce poème s'est ensuite progressivement effacé pour devenir l'emblème d'un nouveau canon poétique qui peut être récité par des enfants ou chanté lors de cérémonies.

11 Dans le prolongement de cette réflexion sur la transformation des canons, Elara Bertho (chapitre 8) montre comment le roman Sarraounia d'Abdulaye Mamani (Niger) fut l'occasion de briser les canons de l'historiographie coloniale en proposant une héroïne de la résistance au colonialisme sous les traits d'une jeune femme séduisante, libérale, laïque, véritable étendard des idéaux socialistes de son auteur. Toutefois, le statut canonique acquis par le roman (qui influence même le contenu des manuels d'histoire) se trouve à présent transformé par des reprises chantées de l'œuvre qui insèrent des problématiques du Niger contemporain sur la place des femmes dans la société ou le rapport entre islam et animisme.

12 Sur les rives du fleuve Sénégal, le canon du Pekaan est indissociable du poète Guélâye Âli Fall. Il se distingue par sa voix exceptionnelle, son sens du rythme et de la musicalité ainsi que par la création de héros et de performances épiques. Marie Lorin montre que de nos jours, si la référence à Guélâye Âli Fall reste indispensable pour légitimer une entreprise littéraire, un nouveau canon se construit avec une poésie principalement géographique qui soulève les enjeux politiques du droit des populations locales sur leur environnement.

Le chapitre 10 propose un questionnement de Ndiabou Séga Touré sur le taasu, un genre chanté et dansé du Sénégal qui exprime la joie et qui s'utilise dans des cérémonies ou pour attirer le chaland lors de la vente à la criée. Ici, c'est la 
transformation du contexte d'utilisation du taasu par sa médiatisation dans la publicité ou dans les chansons de mbalax qui révèle l'importance du rythme comme principal canon du taasu, celui qui détermine tous les autres.

14 Jean Derive analyse ensuite des situations de conflit entre les règles de la linguistique - de la grammaire - et les règles canoniques du genre du discours. Il montre que tant sur le plan de la phonologie que sur celui de la syntaxe ou de la morphologie, les contraintes culturelles liées au formatage canonique peuvent prendre le pas sur les contraintes grammaticales.

Les deux derniers chapitres pourraient constituer une dernière partie de l'ouvrage que l'on pourrait intituler "quand l'anti-canon devient canon ». Marie-Clémence Adom (chapitre 12) étudie le zouglou, une poésie urbaine de Côte d'Ivoire qui revendique l'anti-norme et se démarque des productions précédentes par la critique des "autorités", l'usage unificateur du français, l'emploi des langues locales indépendamment de l'origine du chanteur et enfin la création d'un groupe social qui clame sa marginalité. Ce positionnement hostile à toute forme de norme fait dire à l'auteur que «le canon esthétique du zouglou réside dans la destruction de tous les canons et dans l'érection d'un canon sans cesse en mouvement.» (p. 305). Enfin au dernier chapitre, Kristin Vold Lexander s'interroge sur l'émergence d'une forme canonique dans les pratiques du sms au Sénégal. Analysant la constitution d'un canon avec les outils de l'anthropologie linguistique, elle montre comment une nouvelle norme se crée par des procédés d'enregisterment ${ }^{2}$ et de language policing. Elle montre ainsi comment les choix orthographiques des jeunes et leurs usages des langues deviennent des modèles du texto. Aussi, si l'écriture texto est souvent dépréciée, écrire en bon français comme on écrirait une lettre n'est pas conforme aux canons du sms. Cette anti-norme de l'écriture doit permettre à l'auteur de sms d'exprimer sa créativité et son identité tout en restant compréhensible pour le récepteur de son message.

Outre les analyses précieuses sur ces différents "genres de discours » en Afrique, cet ouvrage propose à mon sens deux apports majeurs. Premièrement, une approche de la parole et des productions de littérature orale soucieuse non seulement du contexte et de la performance mais également des différentes interactions entre l'énonciateur, les énonciataires et les règles canoniques du discours. Ces contributions nous éclairent sur la façon dont la validité canonique d'un discours se constitue et comment une nouvelle forme de prise de parole peut transformer les règles canoniques préexistantes. L'énonciateur doit montrer sa capacité (son talent) à réaliser un énoncé qui correspond en tous points aux règles attendues ou qui, au contraire, transgresse sciemment et si admirablement les règles qu'il en établit de nouvelles. Les énonciataires peuvent approuver une réalisation maitrisée, débattre ardemment du statut et de la légitimité d'une performance transgressive ou, au contraire, adouber une nouvelle forme qui deviendra progressivement un nouveau canon. Enfin, la notion de canons du discours nous apporte un éclairage conceptuel et une approche novatrice des différents enjeux qui président à l'usage juste de la parole. Cet ouvrage s'avère donc précieux pour les chercheurs soucieux d'appréhender les intrications entre parole et relations sociales. Il serait d'ailleurs intéressant de prolonger ces réflexions sur le parler juste en prenant en compte les interactions du quotidien - un aspect délibérément mis de côté dans cet opus-, afin de confronter la notion de canon du discours aux situations d'interlocution et à la façon dont les locuteurs s'emparent, modifient ou subvertissent 
les attentes, en termes de registre et de performance, en fonction du contexte et de l'événement de parole.

\section{NOTES}

1. D. H YMes, "On Communicative Competence ", in J. PRIDE \& J. H OMES (eds.), Sociolinguistics: Selected Readings, Harmondsworth, Penguin, 1972, pp. 53-73.

2. Voir A. AGHA, «Voice, Footing, Enregisterment », Journal of Linguistic Anthropology, 15 (1), 2005, pp. 38-59.

\section{AUTEURS}

\section{NATHANIEL GERNEZ}

Laboratoire d'ethnologie et de sociologie comparative (LESC), Université Paris-Nanterre. 